\title{
Transient global amnesia: Linked to a systemic disorder of amino acid catabolism?
}

\author{
Giuseppe Sancesario • Zaira Esposito • Alessia F. Mozzi • Giulia M. Sancesario • \\ Alessandro Martorana - Angela Giordano $\cdot$ Roberto Sorge $\cdot$ Barbara Mari · \\ Gianfranco Spalletta $\cdot$ Maria Grazia Marciani $\cdot$ Sergio Bernardini
}

Received: 26 January 2013/Revised: 9 April 2013/Accepted: 15 April 2013/Published online: 25 April 2013

(C) The Author(s) 2013. This article is published with open access at Springerlink.com

Dear Sirs,

We aimed to verify whether transient global amnesia (TGA) could be sustained by acute changes of amino acids in the blood, analogous to the transient memory loss induced by experimental tryptophan depletion $[1,2]$.

Plasma amino acids were studied in control healthy subjects (aged 48-65) and in patients (aged 49-70) during the amnesic attack $(n=9)$ and longitudinally at 3 days $(n=12)$ and 16-24 months $(n=9)$ after the amnesic episode, or retrospectively in patients $(n=9)$ who had an amnesic episode 2-3 years before. Routine blood tests for metabolic disorders were also studied. Patients who fulfilled the operational criteria for TGA showed massive impairment of both retrograde and anterograde episodic memory during the acute phase [3-5], but 3-4 days later the memory tests were within the normal range in every case [6]. Brain RMN and EEG examinations showed no relevant abnormalities, even though minimal theta discharges and/or chronic vascular encephalopathy were observed in about half of the patients [5]. Emotional stress and intense physical exertion were reported by $9 / 21$ patients [7]. The study has been approved by the

G. Sancesario $(\bowtie) \cdot$ Z. Esposito · A. Martorana •

A. Giordano · R. Sorge · M. G. Marciani Department of Systems Medicine, Tor Vergata General Hospital, Faculty of Medicine and Surgery, The University of Rome Tor Vergata, 1 Montpellier Street, 00133 Rome, Italy

e-mail: sancesario@med.uniroma2.it

G. Sancesario - A. Martorana - G. Spalletta

Santa Lucia Foundation, Rome, Italy

A. F. Mozzi - G. M. Sancesario - B. Mari - S. Bernardini Department of Experimental Medicine and Surgery, Tor Vergata General Hospital, Faculty of Medicine and Surgery,

The University of Rome Tor Vergata, Rome, Italy appropriate ethics committee and has therefore been performed in accordance with the ethical standards laid down in the 1964 Declaration of Helsinki and its later amendments. All persons gave their informed consent prior to their inclusion in the study.

Blood tests were free from abnormalities, but lactate dehydrogenase (LDH) and aspartate aminotransferase (AST), respectively involved in anaerobic glycolysis and in amino acid metabolism, came out to be constantly overexpressed during, as well as shortly and long after, the amnesic attack (Fig. 1). The levels of plasma amino acids were similar in controls and in patients with a current TGA attack (Table 1). However, the values of glutamine and $\mathrm{L}_{-}$ alanine, but not of the other amino acids, were significantly lower in patients at 3 days (T1) and at 16-24 months (T2) from the amnesic episode compared to age-matched healthy control subjects (Fig. 1). Furthermore, levels of glutamine $(p<0.05)$ and L-alanine $(p<0.01)$ were significantly lower in patients recruited for the study of plasma amino acids 2 to 3 years after a previous TGA episode, compared to controls (Fig. 1). Note that the significant difference between control subjects and patients with a previous TGA episode is enhanced by associating the co-occurrence of low levels of glutamine and L-alanine in the latter group $(p<0.0001)$. To evaluate how the small sample sizes influence the results in our study we performed power analysis. Analysis of group sample sizes of nine for values of glutamine of control healthy subjects and of patients with a previous TGA episode achieved $90 \%$ power to detect a difference of 142.0 between the null hypothesis that both group means are 751.0 and the alternative hypothesis that the mean of TGA group is 609.0 with known group standard deviations of 82.0 and 107.0, respectively, and with a significance level (alpha) of 0.05 , using a $t$ test and assuming that the actual distribution is normal. However, the apparent 

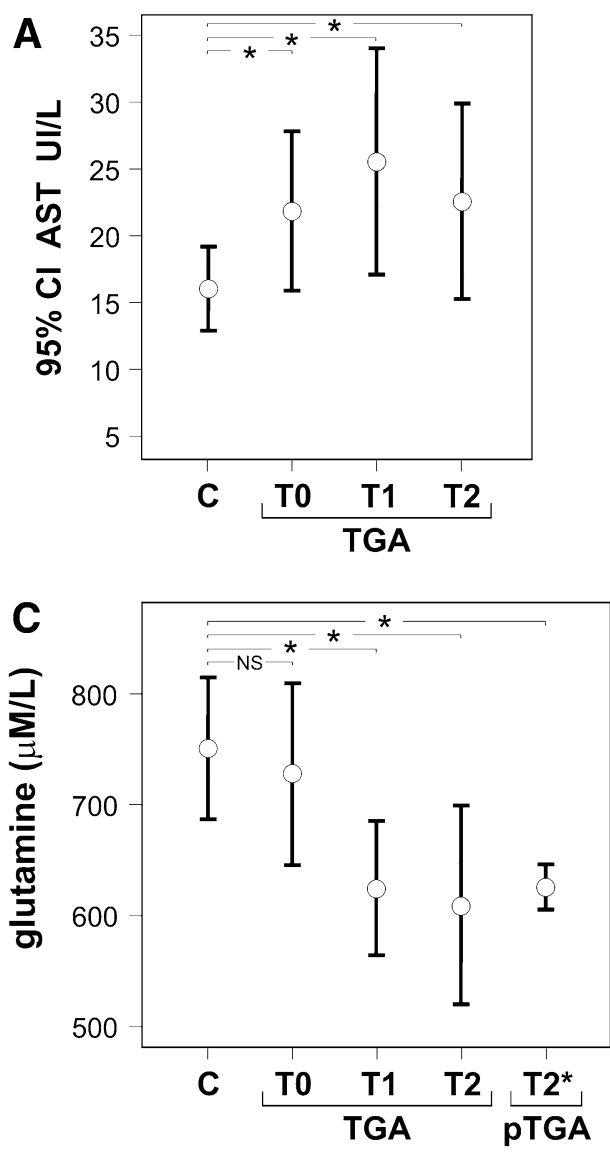

Fig. 1 a total activities (UI/L) of aspartate aminotransferase (AST), and b lactate dehydrogenase (LDH) in blood of normal subjects $(n=9)$ and patients suffering from an episode of transient global amnesia (TGA), studied during the amnesic attack (TGA-T0) $(n=9)$, and at 3 days (TGA-T1) $(n=12)$, and 16-24 months (TGA-T2) $(n=9)$ after the amnesic episode, c concentrations (mean $\pm \mathrm{SD}, \mu \mathrm{M} / \mathrm{L}$ ) of free amino acid glutamine, and $\mathbf{d} \mathrm{L}$-alanine in plasma of normal subjects $(n=9)$ and

increase of glutamine and L-alanine during a TGA attack was highly variable, thus the differences from $\mathrm{T} 0$ to $\mathrm{T} 1-\mathrm{T} 2$ were not significant within the TGA group (Fig. 1). Such non-significant increase of glutamine and L-alanine had not been influenced by an undetermined food intake prior of the amnesic attack, since the values of 1-methylhistidine and 3-methylhistidine, considered an index of daily protein ingestion [8], were similar in the blood samples obtained at the time of the amnesic attack and long after the amnesic episode in the same patients (Table 1). These data suggest that the nonsignificant increase of glutamine and L-alanine during the amnesic attack should be linked to the variable duration of the transient amnesic attack, which may last from 15 min to 24 h. [4].

How can the low steady state of glutamine and L-alanine be interconnected to the overexpression of AST and LDH detected in patients after TGA? LDH catalyzes the interconversion of pyruvate to lactate during anaerobic glycolysis in skeletal muscle, while AST catalyzes the
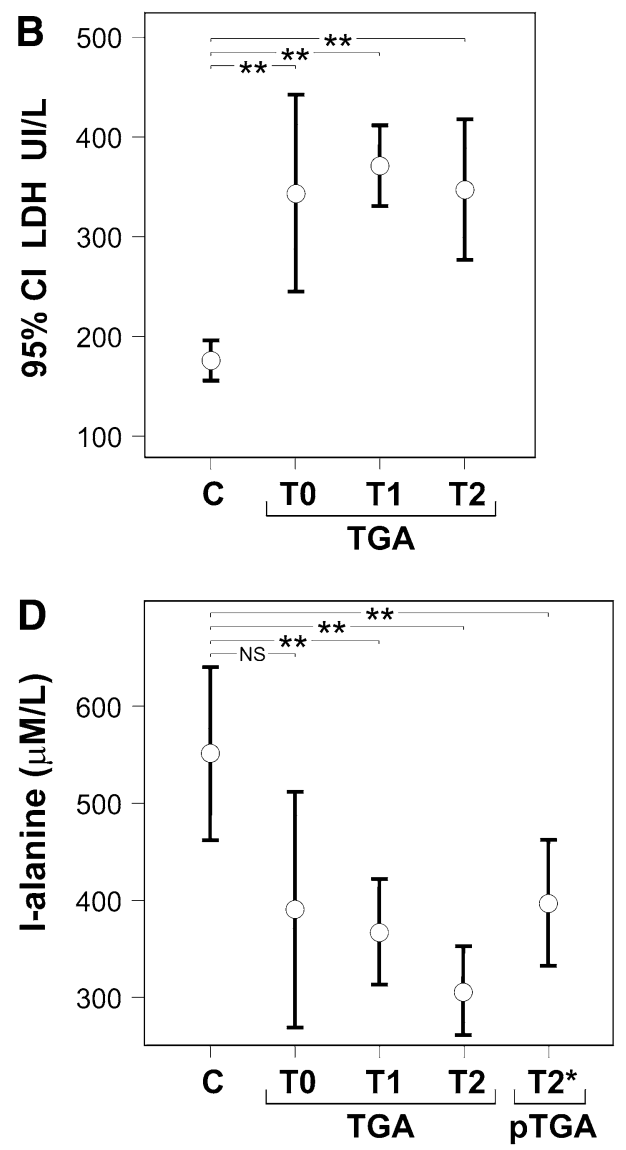

patients suffering from an episode of transient global amnesia, studied either prospectively during the amnesic attack (TGA-T0) $(n=9)$, and at 3 days (TGA-T1) $(n=12)$, and 16-24 months (TGA-T2) $(n=9)$ after the amnesic episode, or retrospectively in a second group of patients that had experienced a previous amnesic episode (pTGA-T2*) 2-3 years before $(n=9)$. One-way ANOVA followed by Bonferroni's test, n.s. nonsignificant, $* p<0.05, * * p<0.01$

interconversion of aspartate and $\alpha$-ketoglutarate to glutamate and oxaloacetate. In turn, glutamate, through the action of alanine aminotransferase, can transfer its amino group to pyruvate, a product of muscle glycolysis, forming L-alanine; moreover, glutamine is synthesized by the enzyme glutamine synthetase, adding a second amino group to glutamate $[9,10]$. Glutamate and glutamine are the most abundant amino acids in the brain: the CSF/plasma ratio for glutamine is indeed more than 0.90 , whereas the concentrations of all other amino acids in CSF are $\sim 10 \%$ or less than the plasma concentrations $[11,12]$. An undue increase of glutamine and L-alanine in the blood may impair their clearance from the brain and the homeostasis of glutamate precipitating the amnesic attack. It is worth noting that the liberation of excitotoxic neurotransmitters in the brain, specifically glutamate, has been suggested as the pathogenic mechanism of TGA [13]. Moreover, MR spectroscopy demonstrated a lactate peak in the hippocampus of patients with TGA, indicating an acute metabolic stress of CA-1 
Table 1 Concentrations of free plasma amino acids

\begin{tabular}{lcccccc}
\hline Amino acid & Control subjects & T0-TGA & T1-TGA & T2-TGA & T2*-pTGA & $p$ \\
\hline b-alanine & $9.536 \pm 4.062$ & $7.189 \pm 3.234$ & $10.380 \pm 4.646$ & $10.000 \pm 0.756$ & $11.182 \pm 5.820$ & n.s. \\
L-alanine & $540.22 \pm 103.48$ & $419.43 \pm 106.36$ & $368.00 \pm 86.02$ & $307.38 \pm 54.74$ & $397.44 \pm 84.34$ & $<0.01$ \\
Glutamine & $751.33 \pm 82.93$ & $727.86 \pm 88.78$ & $625.08 \pm 95.17$ & $609.50 \pm 107.23$ & $625.89 \pm 26.38$ & $<0.05$ \\
Isoleucine & $84.04 \pm 23.85$ & $82.57 \pm 26.77$ & $78.08 \pm 21.27$ & $74.25 \pm 14.69$ & $72.43 \pm 22.71$ & n.s. \\
Leucine & $141.40 \pm 44.69$ & $149.46 \pm 46.05$ & $145.83 \pm 29.14$ & $138.88 \pm 18.92$ & $131.92 \pm 42.75$ & n.s. \\
1-methylhistidine & $7.00 \pm 9.90$ & $5.32 \pm 4.76$ & $5.60 \pm 4.55$ & $7.00 \pm 6.85$ & $9.29 \pm 11.87$ & n.s. \\
3-methylhistidine & $7.87 \pm 8.16$ & $5.97 \pm 2.49$ & $4.93 \pm 2.80$ & $3.63 \pm 1.92$ & $5.11 \pm 1.11$ & n.s. \\
Methionine & $28.91 \pm 7.32$ & $25.46 \pm 4.77$ & $26.83 \pm 5.00$ & $34.00 \pm 5.21$ & $26.03 \pm 4.24$ & n.s. \\
Phenylalanine & $69.17 \pm 15.46$ & $69.69 \pm 13.35$ & $72.66 \pm 31.51$ & $64.75 \pm 12.59$ & $63.20 \pm 10.88$ & n.s. \\
Sarcosine & $5.96 \pm 1.56$ & $4.19 \pm 1.95$ & $5.71 \pm 3.63$ & $4.38 \pm 0.91$ & $4.54 \pm 0.90$ & n.s. \\
Tryptophan & $50.47 \pm 8.22$ & $45.63 \pm 8.76$ & $44.14 \pm 12.11$ & $44.25 \pm 7.96$ & $49.16 \pm 10.7$ & n.s. \\
Tyrosine & $74.50 \pm 26.11$ & $70.89 \pm 13.86$ & $68.41 \pm 6.36$ & $71.00 \pm 9.27$ & $73.81 \pm 27.54$ & n.s. \\
Valine & $266.44 \pm 35.76$ & $254.71 \pm 77.88$ & $257.92 \pm 41.82$ & $250.63 \pm 26.91$ & $241.44 \pm 66.21$ & n.s. \\
Tryptophan/LNAA ratio & $0.078 \pm 0.017$ & $0.073 \pm 0.017$ & $0.069 \pm 0.018$ & $0.070 \pm 0.010$ & $0.0829 \pm 0.018$ & n.s. \\
\hline
\end{tabular}

Concentrations $(\mu \mathrm{M} / \mathrm{L})$ of free amino acids (mean $\pm \mathrm{SD})$ in plasma of normal control subjects $(n=9)$ and of patients with transient global amnesia (TGA) $(n=21)$. Patients with TGA were studied either prospectively during the amnesic attack (T0-TGA) $(n=9)$, at 3 days $($ T1TGA) $(n=12)$, and at 16-24 months (T2-TGA) $(n=9)$ after the amnesic episode, or retrospectively in a second group of patients $(n=9)$ that had suffered a previous amnesic attack (T2*-pTGA) 2-3 years before

Comparisons between groups and within groups n.s. nonsignificant, ${ }^{*} p<0.05$, $* * p<0.01$ glutamine and L-alanine control subjects versus T1TGA, T2-TGA, T2*-pTGA

neurons in these patients [14]. In conclusion, although TGA is by definition a transient and reversible clinical phenomenon lasting no more than $24 \mathrm{~h}$, patients who have undergone a previous TGA episode are characterized by permanent low levels of glutamine and L-alanine and by high levels of AST and LDH in blood, which probably represent constitutive metabolic traits distinctive from that of control subjects. Further research has to be done to evaluate whether such metabolic factors may occasionally predispose to unbalanced catabolism of amino acids and to high levels of glutamate, sustaining a TGA attack.

Acknowledgments The work was supported by Italian Ministry of Health grants RF08.32 (G. Sa.), and RC 10 (G. Sp.). We thank Amanda Formosa, Ph.D., for volunteer editing and comments that improved the manuscript.

Conflicts of interest On behalf of all authors, the corresponding author states that there is no conflict of interest.

Open Access This article is distributed under the terms of the Creative Commons Attribution License which permits any use, distribution, and reproduction in any medium, provided the original author(s) and the source are credited.

\section{References}

1. Riedel WJ, Klaassen T, Schmitt JA (2002) Tryptophan, mood, and cognitive function. Brain Behav Immun 16:581-589
2. Mendelsohn D, Riedel WJ, Sambeth A (2009) Effects of acute tryptophan depletion on memory, attention and executive functions: a systematic review. Neurosci Biobehav Rev 33:926-952

3. Caplan LB (1985) Transient global amnesia. In: Vinken PJ, Bruyn GW, Klawans HL (eds) Handbook of clinical neurology, vol 45. Elsevier, Amsterdam, pp 205-218

4. Hodges JR, Warlow CP (1990) The aetiology of transient global amnesia. A case-control study of 114 cases with prospective follow-up. Brain 113:639-657

5. Pantoni L, Bertini E, Lamassa M, Pracucci G, Inzitari D (2005) Clinical features, risk factors, and prognosis in transient global amnesia: a follow-up study. Eur J Neurol 12:350-356

6. Guillery-Girard B, Desgranges B, Urban C, Piolino P, de la Sayette V, Eustache F (2004) The dynamic time course of memory recovery in transient global amnesia. J Neurol Neurosurg Psych 75:1532-1540

7. Fisher CM (1982) Transient global amnesia. Precipitating activities and other observations. Arch Neurol 39:605-608

8. Stein WH, Beam AG, Moore S (1954) The amino acid content of the blood and urine in Wilson's disease. J Clin Invest S3: 410-419

9. Garber AJ, Karl IE, Kipnis DM (1976) Alanine and glutamine synthesis and release from skeletal muscle. II. The precursor role of amino acids in alanine and glutamine synthesis. J Biol Chem 251:836-843

10. Stumvoll M, Perriello G, Meyer C, Gerich J (1999) Role of glutamine in human carbohydrate metabolism in kidney and other tissues. Kidney Int 55:778-792

11. Hawkins RA, O'Kane RL, Simpson IA et al (2006) Branchedchain amino acids: metabolism, physiological function, and application structure of the blood-brain barrier and its role in the transport of amino acids. J Nutr 136:218S-226S

12. Xiang J, Ennis SR, Abdelkarim GE et al (2003) Glutamine transport at the blood-brain and blood-cerebrospinal fluid barriers. Neurochem Int 43:279-288 
13. Pantoni L, Lamassa M, Inzitari D (2000) Transient global amnesia: a review emphasizing pathogenic aspects. Acta Neurol Scand 102:275-283
14. Bartsch T, Deuschl Gr (2010) Transient global amnesia: functional anatomy and clinical implications. Lancet Neurol 9:205-214 